\title{
Les multiples facettes du genre en médecine
}

\author{
Carole Clair \\ Dr méd., PD, MSc, Médecine adjointe, Policlinique Médicale Universitaire, Lausanne
}

\begin{abstract}
La question du genre en médecine touche diverses sphères. Il peut s'agir de la place des femmes dans la profession médicale, des pathologies spécifiques aux femmes (il s'agit là plutôt de médecine des femmes que de genre, thème que nous n'aborderons pas ici) ou encore des disparités en santé entre les hommes et les femmes.
\end{abstract}

Parlons d'abord du genre en lien avec l'égalité des chances dans la profession médicale. Le domaine médical s'est progressivement féminisé, avec actuellement $60 \%$ d'étudiantes en moyenne dans les facultés de médecine de Suisse. Ceci illustre des normes sociales qui ont progressivement changé: l'accès aux études de médecine est non seulement devenu possible pour les femmes suisses à partir de la fin du XIX ${ }^{\mathrm{e}}$ siècle - la première femme médecin suisse, Marie Heim Vögtlin, a débuté ses études en 1868 - mais est maintenant socialement accepté. Ceci étant, on compte pourtant moins de $20 \%$ de femmes professeures dans ces mêmes universités (ce chiffre étant plus proche de 10-15\% dans les facultés de médecine) [1], ce qui n’est donc pas

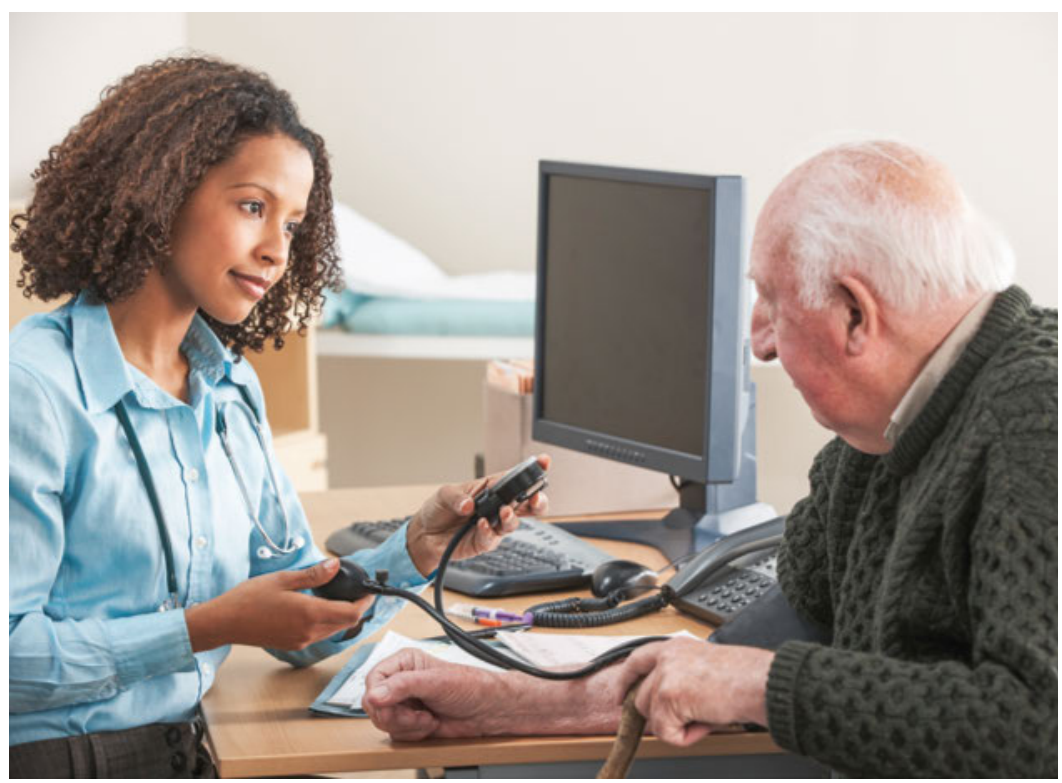

Le domaine médical s'est progressivement féminisé, avec actuellement 60\% d'étudiantes en moyenne dans les facultés de médecine de Suisse. Ceci étant, on compte pourtant moins de $20 \%$ de femmes professeures dans ces mêmes universités. un problème de proportionnalité, mais résulte bien d'un phénomène qui empêche les femmes d'accéder au sommet de la hiérarchie, le fameux plafond de verre. A cela s'ajoutent les parois de verre, qui font que les femmes sont plus facilement dirigées vers des professions ou secteurs moins prestigieux qui ne vont pas permettre leur ascension professionnelle.

Le deuxième thème est celui des disparités de genre en médecine. Une médecine longtemps androcentrée - prenant comme référence le jeune homme blanc a négligé les autres catégories, et notamment les femmes. Or, on sait qu'il existe des différences métaboliques, hormonales, physiologiques, entre les hommes et les femmes, et qu'il est important d'en tenir compte. Ceci, même sans céder aux stéréotypes de genre qui conduisent à des différences de prise en charge non justifiées entre les hommes et les femmes. Un exemple est la prise en charge de la douleur. Des études ont démontré que les femmes attendent 15 minutes de plus avant de recevoir un traitement antalgique aux urgences lorsqu'elles se présentent avec des douleurs abdominales [2]. Lorsqu'elles sont prises en charge, le traitement est moins agressif, les hommes étant plus susceptibles de recevoir des traitements à base d'opiacés. A l'inverse, la dépression est sous-diagnostiquée chez les hommes, victimes du stéréotype selon lequel l'homme est fort et résistant, avec comme corollaire des diagnostics manqués et un taux de suicide quatre fois plus élevé [3].

A ces thèmes s'ajoute le harcèlement, qui malheureusement n'épargne pas le monde médical. Ce dernier peut être frontal mais peut aussi prendre des formes plus insidieuses. Le sexisme ordinaire, qui consiste en des allusions sexistes, des propos paternalistes ou condescendants, se retrouve particulièrement dans les 
milieux où les femmes sont rares. Il opère souvent sous couvert de l'humour. Heureusement les langues se délient aujourd'hui notamment via les réseaux sociaux, avec des sites comme "Paye ta blouse» où

\section{Une médecine longtemps androcentrée - prenant comme référence le jeune homme blanc.}

femmes (et hommes) dénoncent les comportements dégradants envers les femmes dans le milieu médical. Comment aller de l'avant? Il ne suffit pas de critiquer ou dénoncer les inégalités de genre, il faut les corriger. Pour ceci, l'historienne des sciences, Londa Schiebinger, propose d'agir sur trois fronts selon le slogan: «Fix the women - Fix the institutions - Fix the knowledge» [4].

Il faut que les femmes se mettent en avant, osent s'imposer. Les programmes de mentoring, de formation personnelle, et la présence de modèles féminins en médecine, peuvent aider à ce que les femmes brisent le plafond de verre. Mais cela ne suffit malheureusement pas. Des études ont démontré qu'à compétences égales, et même sans charges familiales, les femmes n'ont pas les mêmes chances de promotion que les hommes. Il faut donc travailler au niveau institutionnel. Lutter contre les biais inconscients lors du recrutement qui font qu'une candidature masculine va être favorisée. Faire en sorte que les facultés s'engagent pour promouvoir des femmes aux postes clés et fournissent un environnement favorable pour permettre aux femmes de mener de front leur carrière. Il faut aussi mobiliser les hommes, parvenir à une répartition des tâches familiales ou domestiques plus égalitaire, et favoriser le temps partiel chez les hommes qui le souhaitent. Bref essayer de faire bouger le modèle classique de l'homme à $150 \%$ et de la femme à $50 \%$.

Finalement, il faut poursuivre les recherches sur les biais de genre en santé, déterminer ce qui est lié au domaine biologique (le sexe) de ce qui est de l'ordre social (le genre). Il faut enseigner le genre en médecine et sensibiliser la relève à la problématique du genre qui est transversale à toutes les disciplines et spécialités.

Récemment, le Forum économique mondial a publié le classement des pays en terme d'égalité hommesfemmes, en se basant sur un indice comprenant no-

\section{forumsante.ch}

Le mardi 23 janvier à I'Hôtel Bellevue à Berne, forumsante.ch organise pour la $19^{e}$ fois une journée de réflexion sur un thème d'actualité: "Les femmes à la conquête du système de santé: quelles conséquences?». Des conférencières suisses et internationales de haut niveau traiteront des aspects sociétaux, économiques, politiques et éthiques de cette question.

forumsante.ch ne se limite pas à discuter ce qui est, mais s'attache bien davantage à envisager et à réfléchir l'avenir - on y expose, construit et échange des idées pointues et originales sur le futur.

Site Internet (programme et inscription): www.forumsante.ch

tamment les inégalités salariales, l'accès à la santé et à l'éducation ou les représentations politiques. Selon ce rapport, la Suisse régresse et se trouve au $21^{\mathrm{e}}$ rang, derrière l'Islande, la France ou le Nicaragua. Ce même rapport estime les gains financiers pour le PIB d'un pays si cette égalité est atteinte, et ils se chiffrent en centaine de milliards de francs. En améliorant la qualité des

\section{Il ne suffit pas de critiquer ou dénoncer les inégalités de genre, il faut les corriger.}

soins, grâce à une approche sensible au genre, et en éliminant les inégalités de genre en santé - pour les patient-e-s mais également pour les professionnel-le-s de santé -, nous arriverons non seulement à améliorer le fonctionnement du système de santé, mais peut-être aussi à apporter des gains économiques et surtout offrir une prise en charges adaptée et de qualité aux patientes et aux patients.

\section{Crédit photo}

(C) Monkeybusinessimages | Dreamstime.com

\section{Références}

1 Programme CUS 2013-2016 P-4 «Egalité des chances entre Femmes et Hommes dans les Universités/Etudes genre». https://www. swissuniversities.ch/fr/themes/encouragement-de-la-releve/ egalite-des-chances/programme-cus-p-4/.

2 Chen EH, Shofer FS, Dean AJ, et al. Gender disparity in analgesic treatment of emergency department patients with acute abdominal pain. Acad Emerg Med. 2008;15(5):414-8.

3 Moller-Leimkuhler AM. The gender gap in suicide and premature death or: why are men so vulnerable? Eur Arch Psychiatry Clin Neurosci. 2003;253(1):1-8.

4 Schiebinger L. Gendered Innovations. http://genderedinnovations.stanford.edu/what-is-gendered-innovations.html, 2017 